\title{
On the wind noise reduction mechanism of porous microphone windscreens
}

\author{
Sipei Zhao, a) Matthew Dabin, and Eva Cheng \\ School and Engineering, RMIT University, Melbourne, Australia \\ Xiaojun Qiu and lan Burnett \\ Centre for Audio, Acoustics and Vibration, Faculty of Engineering and IT, University of Technology Sydney, \\ Sydney, Australia \\ Jacob Chia-chun Liu \\ Department of Water Resources and Environmental Engineering, Tamkang University, Taiwan
}

(Received 25 July 2017; revised 22 September 2017; accepted 10 October 2017; published online 30 October 2017)

\begin{abstract}
This paper investigates the wind noise reduction mechanism of porous microphone windscreens. The pressure fluctuations inside the porous windscreens with various viscous and inertial coefficients are studied with numerical simulations. The viscous and inertial coefficients represent the viscous forces resulting from the fluid-solid interaction along the surface of the pores and the inertial forces imposed on the fluid flow by the solid structure of the porous medium, respectively. Simulation results indicate that the wind noise reduction first increases and then decreases with both viscous and inertial coefficients after reaching a maximum. Experimental results conducted on five porous microphone windscreens with porosity from 20 to 60 pores per inch (PPI) show that the 40 PPI windscreen has the highest wind noise reduction performance, and this supports the simulation results. The existence of the optimal values for the viscous and inertial coefficients is explained qualitatively and it is shown that the design of the porous microphone windscreens should take into account both the turbulence suppression inside and the wake generation behind the windscreen to achieve optimal performance. (C) 2017 Acoustical Society of America. https://doi.org/10.1121/1.5008860
\end{abstract}

[DDE]

Pages: 2454-2463

\section{INTRODUCTION}

Microphone windscreens have been widely used in outdoor noise and ventilation system acoustic measurements to minimize the effect of wind noise (Larsson et al., 2005; Shams et al., 2005). Although this measure for reducing wind noise is well known and widely used, the physical mechanisms of the wind noise reduction by microphone windscreens has not been fully understood.

Strasberg (1988) investigates the wind noise sensed by microphones in spherical and cylindrical windscreens and finds that the wind noise levels measured by different authors with different windscreens form a single curve when plotted against the Strouhal number $(f D / U$, where $f$ is the frequency, $D$ is the windscreen diameter, and $U$ is the mean wind speed). It is shown that the wind noise level decreases linearly with the dimensionless frequency in log-scale for values of $f D / U$ up to 5 (Strasberg, 1988). Morgan and Raspet (1992) point out that Strasberg's analysis is only valid for low-turbulence environments because the data used by Strasberg is measured in laboratories by moving the screened microphones through substantially quiet air. In contrast, the dominant source of pressure fluctuations at the microphone outdoors is the intrinsic turbulence in the flow, hence the optimum design of windscreens for outdoor measurements may require consideration of factors other than

\footnotetext{
a)Electronic mail: sipeizhao@sina.com
}

wake generation and reduction of flow through the windscreen (Morgan and Raspet, 1992).

Raspet et al. (2006) propose a method for predicting the upper and lower bounds of wind noise measured in spherical windscreens from the measured incident velocity spectra, and the measurement results in $180 \mathrm{~mm}$ windscreens shows consistency with the theories. The method is further extended to the source region (lower frequency range) of the atmospheric wind turbulence, and outdoor measurements with a large range of windscreen sizes are performed to validate the theory (Raspet et al., 2008).

Yu et al. (2011) measure the wind noise at the ground surface by placing the microphone underneath a thin layer of foam and they also measure the low frequency wind noise above the ground with a microphone inside spherical fiberglass windscreens. A thin layer of foam is used in outdoor wind noise measurements by Raspet and Webster (2015) to eliminate the effect of the stagnation interaction pressure on the microphones. Spherical microphone windscreens are also used for outdoor measurements above flat open grassland by Boersma (1997). van den Berg (2006) proposes that the windscreens could be treated as a first order low pass filter for pressure fluctuations due to atmospheric turbulence based on an analysis of the outdoor measurement results from different authors.

In addition to the abovementioned studies for outdoor noise measurement, the wind noise reduction effects of windscreens are also investigated in wind tunnels. Lin et al. (2014) 
measure the wind noise reduction with different types of windscreens in the low frequency range from 20 to $200 \mathrm{~Hz}$, showing that the noise reduction performances of various types of windscreens are similar when the wind speed is under $1.5 \mathrm{~m} / \mathrm{s}$; however, for wind speeds above $2.0 \mathrm{~m} / \mathrm{s}$, the noise reduction of the single layer fabric windscreen $(40 \mathrm{~cm}$ diameter) is much better than the spherical windscreens ( 7 and $20 \mathrm{~cm}$ diameter) and double layer $(30$ and $40 \mathrm{~cm}$ diameter) windscreens.

Wang et al. (2012) measure the self-noise of microphone windscreens in an anechoic wind tunnel and found that the wind noise is generally more effectively attenuated by windscreens with larger diameters, but windscreens with diameter 60 and $90 \mathrm{~mm}$ show similar performance. Alamshah et al. (2015) investigate the effects of turbulent flow characteristics on wind noise generation in microphone windscreens, and find that the wind noise at very low frequencies increases with the average turbulent length scales while the overall wind noise inside windscreens is insensitive to the incoming flow turbulence intensity. Raspet et al. (2014) measure the correlation length of pressure fluctuations inside a $180 \mathrm{~mm}$ porous microphone windscreen and find that the wind noise reduction mechanism by microphone windscreens is attributed to the spatial decorrelation of the pressure fluctuations.

Although many different windscreens are used in both indoor and outdoor measurements, the noise reduction mechanism of windscreens still lacks theoretical analyses. A primarily intuitive explanation is that the windscreens can reduce the wind speed at the microphone, hence reduce the measured wind noise (Phelps, 1938). Phelps (1938) models the windscreen as a rigid sphere and calculated the pressure inside the sphere by averaging the pressure distribution on the spherical surface, with the assumption that the air flow is inviscid, incompressible and irrotational. Zheng and Tan (2003) model the microphone windscreen as a rigid impermeable sphere to investigate the effects of Reynolds number on the wind noise reduction performance. The analytical solutions for the inviscid and Stokes flows are pursued as the two extreme cases for infinite and low Reynolds number flows, respectively, and the intermediate and high Reynolds number flows are studied with a numerical scheme (Zheng and Tan, 2003). These models are simplified because the windscreens are treated as a rigid impermeable sphere, and the air around windscreens are assumed to be steady-state laminar flows, both of which are different from real applications.
Xu et al. (2011) perform a computational study on the effect of windscreen shape and flow resistivity on turbulent wind noise reduction by modeling the windscreen as the porous material. The effects of windscreen shape and the porous material properties (i.e., flow resistivity) are investigated, and it is found that the circular and horizontal ellipse windscreens have similar overall wind noise reduction performance, while the horizontal ellipse windscreen with medium flow resistivity provides larger wind noise reduction (Xu et al., 2011).

This paper investigates the wind noise reduction mechanism of porous microphone windscreens. Different from previous studies that focus on the viscous effect of porous windscreens with different shapes, the numerical simulations in this paper account for both the viscous and inertial forces from the porous windscreens. The pressure fluctuations inside the porous windscreens with various viscous and inertial coefficients are studied, and the simulation results are analyzed and explained. The wind noise reduction by 5 different porous microphone windscreens is measured with a fan to verify the simulations results.

\section{SIMULATION MODEL}

The diagram of the simulation model is shown in Fig. 1(a), where the uniform air flow with mean speed $U$ enters the computation domain from the left boundary. An array of solid cylinders with diameter $D$ is placed upstream to generate turbulence. The solid cylinder array is $4 D$ from the flow inlet boundary and the interval between the solid cylinders is also $D$. The grey circle in the middle denotes the microphone windscreen of diameter $D_{0}$, which is modeled as a porous medium. The computation domain is $34 D$ in the downwind direction and $10 D$ in the crosswind direction, and the microphone windscreen is $10 D$ from the upstream solid cylinder array.

Although the sound waves measured at a microphone are compressible pressure disturbances, the wind noise is actually the turbulent pressure fluctuations (pseudo sound) generated by the incoming flow on the microphone (Xu et al., 2011). In the scenario of acoustic measurement in windy conditions, the wind speed is usually much smaller than the speed of sound, hence the turbulent flow can be approximated as incompressible turbulence. The fluid flow outside the porous microphone windscreen is described by the Navier-Stokes equations for viscous incompressible flow (Xu et al., 2011),

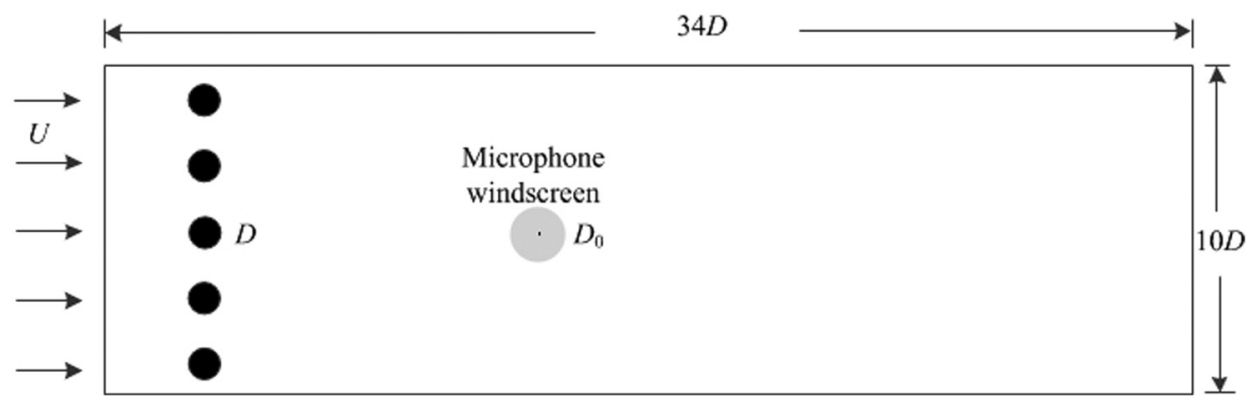

FIG. 1. Diagram of the simulation model. 


$$
\begin{aligned}
& \nabla \cdot \mathbf{u}=0 \\
& \frac{\partial \mathbf{u}}{\partial t}+(\mathbf{u} \cdot \nabla) \mathbf{u}=-\frac{1}{\rho} \nabla p+\nu \nabla^{2} \mathbf{u},
\end{aligned}
$$

where $\mathbf{u}$ is the velocity, $p$ is the pressure, $\rho$ is the fluid density and $\nu$ is the viscosity of the fluid.

The fluid flow inside the porous microphone windscreen is governed by the continuity equation and the momentum conservation equation proposed by Nithiarasu et al. (1997),

$$
\begin{aligned}
& \nabla \cdot \mathbf{u}=0 \\
& \frac{1}{\phi} \frac{\partial \mathbf{u}}{\partial t}+\frac{1}{\phi^{2}}(\mathbf{u} \cdot \nabla) \mathbf{u}=-\frac{1}{\rho} \nabla p+\frac{\nu}{\phi} \nabla^{2} \mathbf{u}-\frac{\nu}{K} \mathbf{u}-C|\mathbf{u}| \mathbf{u}
\end{aligned}
$$

where $\phi$ is the porosity of the porous medium, $\mathbf{u}$ and $p$ are the superficial (volume-averaged) velocity and pressure, respectively, $K$ is the permeability of the porous medium, and $C$ is the inertial coefficient. The derivation of Eq. (4) is based on the volume averaging technique. All the quantities including the velocity, pressure, viscous and inertial coefficients in Eq. (4) are averaged over a representative elementary volume, which is much larger than any individual pores but is much smaller than the whole porous material (the porous windscreen in our case) (Nithiarasu et al., 1997). The advantage of this generalized momentum conservation equation is that it can be reduced to the conventional Navier-Stokes equation when there is no porous media (both the viscous and inertial coefficients are 0 and the porosity is 1 ), so the Navier-Stokes solver can also be used for such equations.

The porous windscreen introduces extra two terms in the momentum conservation equation in Eq. (4) compared to the Navier-Stokes equation for the fluid flow without a porous medium. The third term on the right hand side of Eq. (4) is the Darcy term which represents the viscous forces resulting from the fluid-solid interaction along the surface of the pores in the porous medium. The fourth term on the right hand side of Eq. (4) is the Forchheimer term, which represents the inertial forces imposed on the fluid flow by the solid structure of the porous medium (Narasimhan, 2013).

The physical mechanism of wind noise reduction by porous microphone windscreens was found to be the resistance forces caused by the porous windscreen on the fluid flow to reduce the fluctuation amplitude of the turbulent velocity and pressure (Narasimhan, 2013). The resistance forces consist of the viscous forces resulting from the viscous stresses along the fluid-solid interface of the pores and the inertial forces imposed on the fluid by the solid permeable medium. The viscous forces depend on the fluid viscosity and the permeability of the porous media $K$, while the inertial forces can be characterized by the inertial resistance coefficient $C$, which has the dimensions of length and is characteristic of the geometry of the media, the cell size, and the nature of the flow (Dawson et al., 2007).

Equations (1)-(4) were solved in FLUENT 16.0 by modeling the microphone windscreen as a "porous media zone" with the boundary condition of velocity and stress continuity at the windscreen surface. The models were built, and meshed in ANSYS Workbench 16.0, and simulated in ANSYS FLUENT 16.0. In the simulations, the boundary condition of the flow inlet was set to "velocity inlet," the output boundary condition was set to "pressure outlet," and the upper and lower boundaries were set as "wall." In the simulations performed in this paper, the wind speed at the inlet is $U=4 \mathrm{~m} / \mathrm{s}$, and the diameter of the upstream cylinder and the porous windscreen are $D=50 \mathrm{~mm}$ and $D_{0}=90 \mathrm{~mm}$, respectively. The Reynolds number for the flow in the simulations can be estimated as $\operatorname{Re}=U D / \nu=1.3 \times 10^{4}$ (where $U=4 \mathrm{~m} /$ is the mean flow speed, $D=0.05 \mathrm{~m}$ is the diameter of the upstream cylinders and $\nu=1.511 \times 10^{-5}$ is the air kinematic viscosity at the temperature of $20^{\circ} \mathrm{C}$ ). The Reynolds number based on the Taylor microscale for the turbulent flow in the simulations is about $\operatorname{Re}_{\lambda} \approx(20 \mathrm{Re} / 3)^{1 / 2}=294$.

The velocity and pressure fluctuations at the center of the porous windscreen are monitored because microphones are usually installed in the center of the windscreens in experimental measurements. For each simulation, the time history of velocity and pressure fluctuations at the monitoring location is recorded for $5 \mathrm{~s}$ with a sampling rate of $10 \mathrm{kHz}$. The recorded data were divided into eight sections with $50 \%$ overlap, and the power spectral density of each section was estimated by using the Welch method with the Hamming window. The velocity and pressure spectra were obtained by averaging the power spectral density of the eight sections. It is noteworthy that the numerical simulations in this paper are two dimensional only and the pressure spectrum can be different to that for the three dimensional turbulence. In addition, because the Reynolds number is relatively small, the inertial subrange may not be observed in the current simulations according to the recent numerical and experimental results (Gotoh and Fukayama, 2001; Tsuji and Ishihara, 2003).

To quantitatively examine the wind noise reduction performance of the windscreens, the wind velocity reduction (WVR) and wind noise reduction (WNR) as a function of frequency are defined in Eqs. (5) and (6), respectively,

$$
\begin{aligned}
& \operatorname{WVR}(f)=10 \log _{10} \frac{\left|u_{0}(f)\right|^{2}}{\left|u_{\mathrm{ws}}(f)\right|^{2}}(\mathrm{~dB}), \\
& \mathrm{WNR}(f)=10 \log _{10} \frac{\left|p_{0}(f)\right|^{2}}{\left|p_{\mathrm{ws}}(f)\right|^{2}} \quad(\mathrm{~dB}),
\end{aligned}
$$

where $u_{0}(f)$ and $p_{0}(f)$ are the incompressible turbulent velocity and pressure fluctuation magnitude without windscreen at frequency $f$, and $u_{\mathrm{ws}}(f)$ and $p_{\mathrm{ws}}(f)$ are the corresponding incompressible turbulent velocity and pressure magnitude within windscreens. The overall WNR in a frequency range from between $f_{1}$ and $f_{N}$ is defined as

$$
\mathrm{WNR}=10 \log _{10} \frac{\sum_{f_{1}}^{f_{N}}\left|p_{0}\left(f_{n}\right)\right|^{2}}{\sum_{f_{1}}^{f_{N}}\left|p_{\mathrm{ws}}\left(f_{n}\right)\right|^{2}}(\mathrm{~dB}) .
$$


The effect of the viscous and inertial resistance on the wind noise reduction performance is simulated in Sec. III and the results are explained. It is noteworthy that throughout the text in this paper, the wind velocity and wind noise refer to the incompressible turbulent velocity and pressure fluctuations rather than the compressible acoustic pressure disturbances.

\section{SIMULATION RESULTS}

Because the resistance on the air flow due to the porous windscreen consists of the viscous and inertial forces, the effect of the viscous and inertial forces on the wind noise reduction performance is investigated separately in the two sets of simulations below.

\section{A. The viscous effect}

In the first set of simulations, a zero inertial coefficient was assumed so that no inertial force was applied on the air flow from the porous windscreen. In this case, the physical wind noise reduction mechanism of windscreens results only

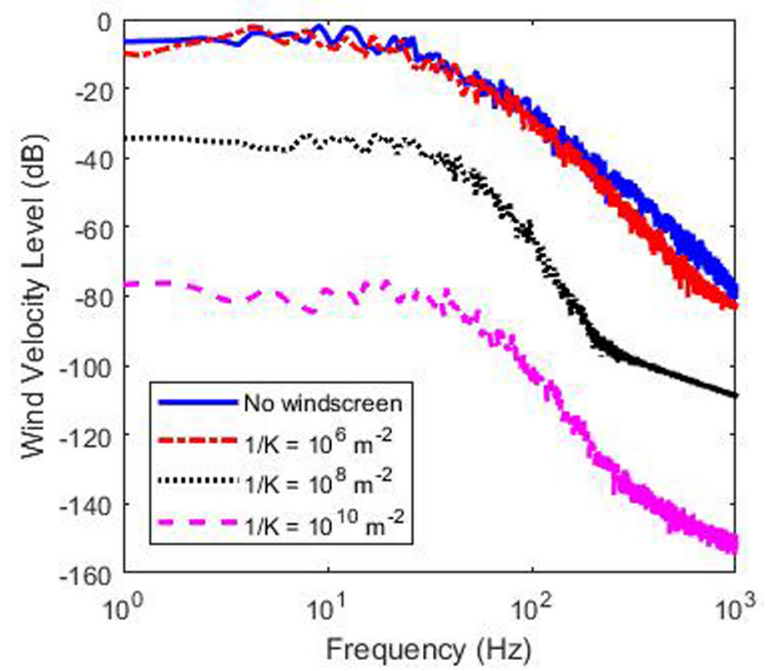

(a)

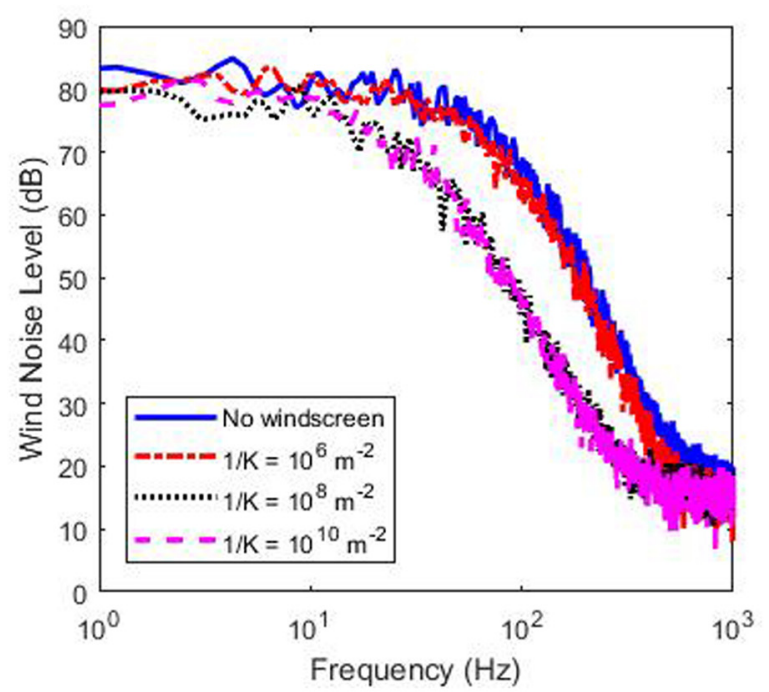

(b)

FIG. 2. (Color online) (a) Wind velocity spectra and (b) wind noise spectra for different viscous coefficients at the wind speed $U=4 \mathrm{~m} / \mathrm{s}$. from the viscous forces on the air flow by the porous windscreen. This is actually the same scenario as the computational study by $\mathrm{Xu}$ et al. (2011), where the flow resistivity is used to characterize the material viscous property. The flow resistivity $\sigma$ is related to the viscous coefficient $1 / K$ ( $K$ is the permeability) by $\sigma=\mu / K$, where $\mu$ is the air viscosity (Xu et al., 2011).

The simulation results of the wind velocity spectra and the wind noise spectra for various viscous coefficients are shown in Fig. 2. The wind velocity and pressure level generally increase with the wind speed, and the wind speed $U=4 \mathrm{~m} / \mathrm{s}$ is used in the simulations for consistency with the experimental conditions. It can be observed from Fig. 2(a) that the wind velocity inside the porous windscreen decreases with growing viscous coefficients $1 / K$. This is reasonable because for small viscous coefficients, the porous windscreen is highly permeable and has little effect on the incoming air flow, whereas the porous windscreen with a large viscous coefficient produces large viscous force on the air flow so the flow speed is damped heavily.

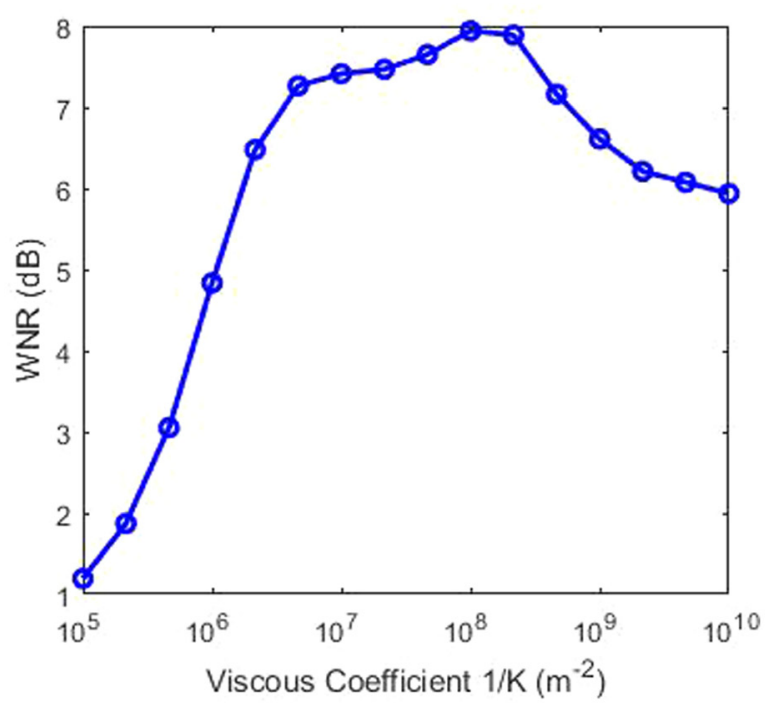

(a)

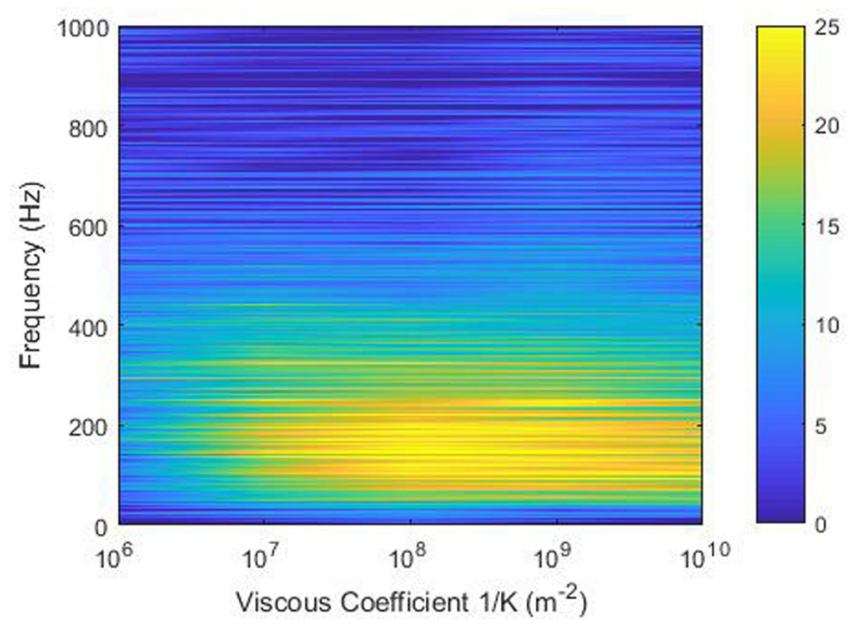

(b)

FIG. 3. (Color online) (a) The overall WNR and (b) the WNR at different frequencies for $90 \mathrm{~mm}$ windscreens with different viscous coefficients at the wind speed $U=4 \mathrm{~m} / \mathrm{s}$. 
In contrast, Fig. 2(b) shows that the wind noise level first decreases then slightly increases with the growing viscous coefficients. These results are consistent with the computational simulation by $\mathrm{Xu}$ et al. (2011), where the windscreens with medium flow resistivity have the most effective wind noise reduction performance. This can be more clearly observed from Fig. 3(a), which shows the overall WNR in a broad frequency range from 1 to $1000 \mathrm{~Hz}$. The WNR reaches its maximum when the viscous coefficient is around $10^{8} \mathrm{~m}^{-2}$, and decreases slightly afterward. This is also illustrated by Fig. 3(b) for the WNR at different frequencies. The viscous coefficient is a quantity for characterizing the viscous forces due to the fluid-solid interaction along the surface of the pores in the porous medium, which is related to the roughness of the pore surface.

The existence of the optimal viscous coefficient for porous windscreens on wind noise reduction can be explained physically. When there is no windscreen, the wind noise is primarily due to the turbulence in the incoming flow from the upstream solid cylinder array, as shown in Fig. 4(a). When the windscreen is present, the wind noise is reduced because the windscreen suppresses the turbulence inside the porous material. For the viscous coefficient below $10^{8} \mathrm{~m}^{-2}$, the larger the viscous resistance, the greater the wind noise reduction, as shown in Figs. 4(b) and 4(c). However, for a viscous coefficient larger than $10^{8} \mathrm{~m}^{-2}$, the windscreen tends to be a solid cylinder and the wake is generated behind the windscreen, which deteriorates the wind noise reduction performance, as shown in Fig. 4(d).

\section{B. The inertial effect}

In the second set of simulations, a zero viscous coefficient was assumed so that no viscous force was applied on the air flow from the porous windscreen. In this case, the physical wind noise reduction mechanism of the windscreens results from the inertial forces on the air flow by the solid frame of the porous windscreen.

The simulated wind velocity spectra and wind noise spectra are shown in Fig. 5 for various inertial coefficients, which demonstrate that the wind velocity inside the porous windscreen decreases with growing inertial coefficients while the wind noise level first decreases then increases with inertial coefficients. Similar to the viscous effect, the WNR reaches its maximum near the inertial coefficient of $50 \mathrm{~m}^{-1}$ and decreases slightly afterward. This is illustrated in Fig. 6 with the overall WNR in the frequency band from 1 to $1000 \mathrm{~Hz}$. This phenomenon can again be explained by the trade-off between the turbulence suppression inside and the wake generation behind the windscreens, as shown in Fig. 7. When the inertial coefficient is smaller than $50 \mathrm{~m}^{-1}$, the turbulence inside the windscreen is suppressed while there is no wake generated behind due to the permeability of the windscreen. When the inertial coefficient is larger than $50 \mathrm{~m}^{-1}$, the windscreen is less permeable and wake is formulated. The inertial coefficient is a characterization of the inertial force on the air flow to change the flow direction, which is dependent on the tortuosity of the porous medium.

By comparing the wind velocity spectra and wind noise spectra in Figs. 2 and 5, it can be observed that the wind velocity level decreases continuously with the viscous or inertial coefficients in the broadband from 1 to $1000 \mathrm{~Hz}$, whereas the wind noise is only reduced in the frequency band below $500 \mathrm{~Hz}$ and has the lowest level at a certain value of viscous or inertial coefficients. The inconsistency between the wind velocity and wind noise inside the windscreens shows that the wind noise level is not proportional to

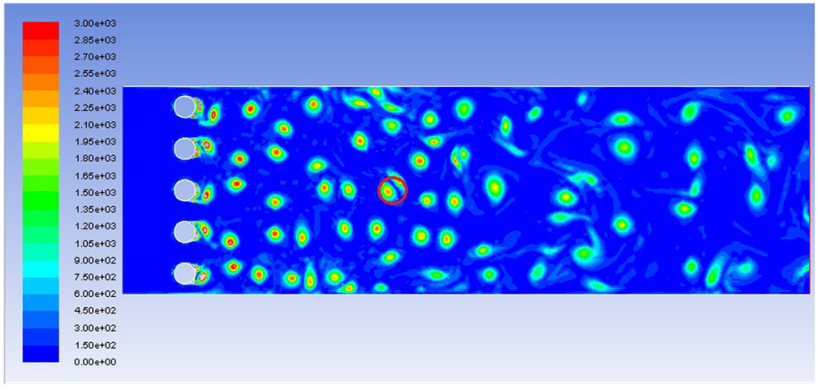

(a)

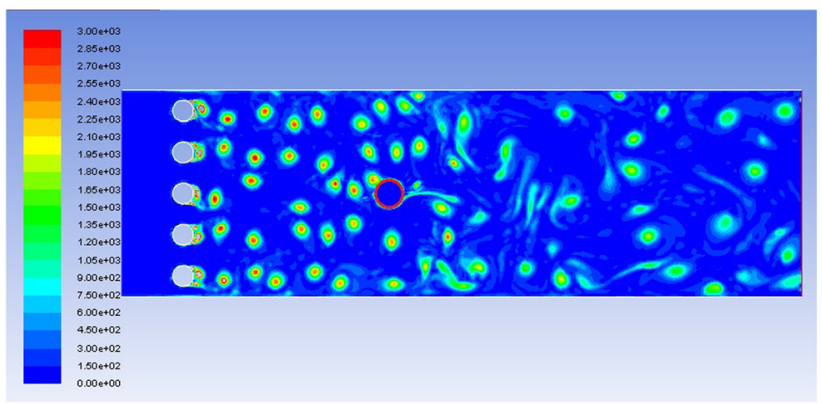

(c)

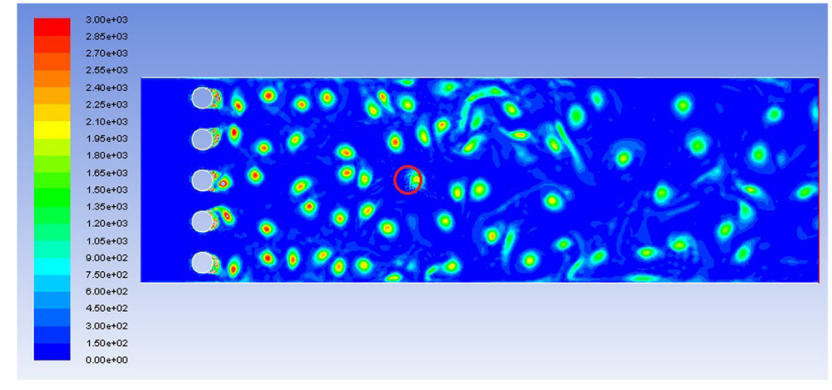

(b)

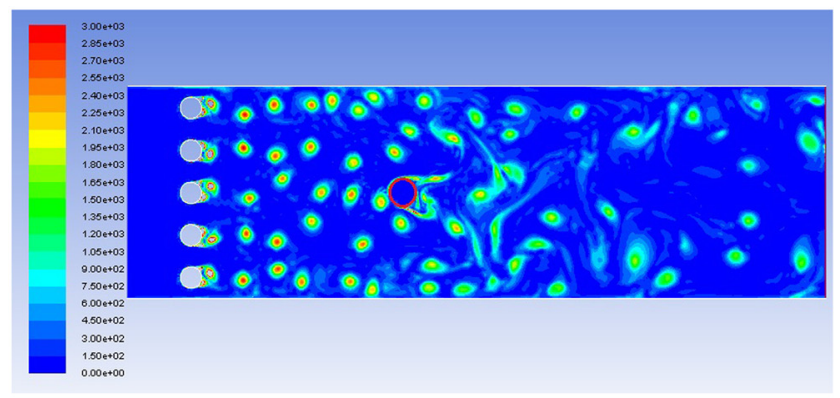

(d)

FIG. 4. (Color online) The vorticity contour for different viscous coefficients, (a) $1 / K=0$ (no windscreen), (b) $1 / K=10^{6} \mathrm{~m}^{-2}$, (c) $1 / K=10^{8} \mathrm{~m}^{-2}$, and (d) $1 / K=10^{10} \mathrm{~m}^{-2}$. The red circle in the middle denotes the location of the microphone windscreen. 


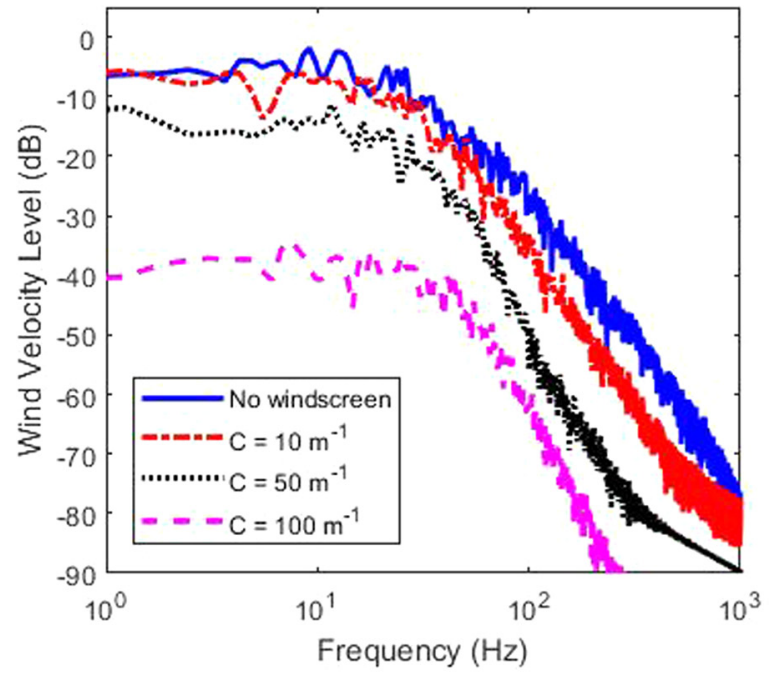

(a)

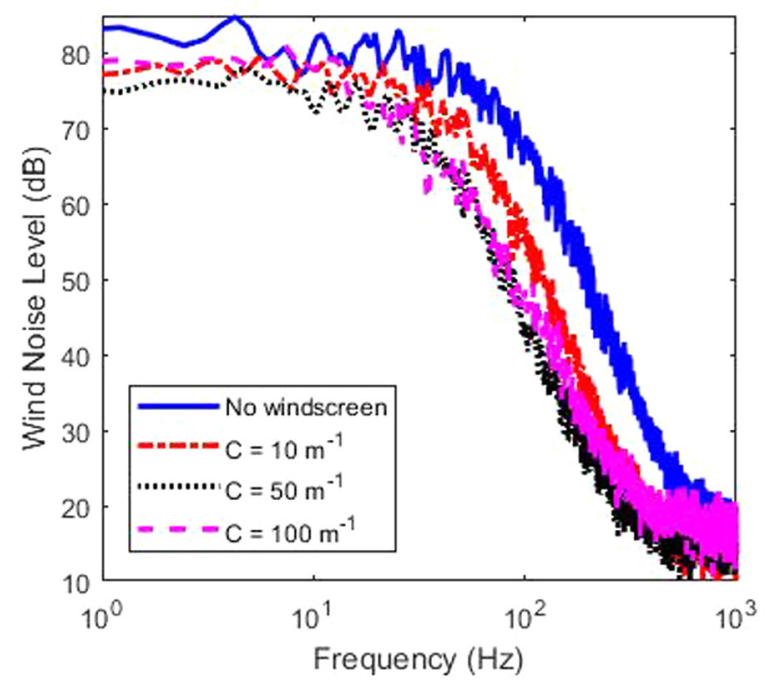

(b)

FIG. 5. (Color online) (a) Wind velocity spectra and (b) wind noise spectra for different inertial coefficients at the wind speed $U=4 \mathrm{~m} / \mathrm{s}$.

the wind velocity inside the porous windscreens, as stated in the intuitive explanation (Phelps, 1938).

\section{The combination effect}

The above simulation results show separately the individual effect of the viscous force or inertial force on the wind noise reduction performance by the porous windscreens. To understand the wind noise reduction performance of real windscreens with both viscous and inertial forces on the air flow, various combinations of the viscous and inertial coefficients are investigated. The simulation results are shown in Fig. 8, where the horizontal and vertical axes are the viscous and inertial coefficients, respectively, and different colors are used to represent different levels of WNR.

It can be observed that the viscous forces are the main source of wind noise reduction mechanism for the porous windscreens when the inertial coefficient is below $50 \mathrm{~m}^{-1}$, while for the inertial coefficient larger than $100 \mathrm{~m}^{-1}$, the viscous forces have little effect. Figure 8 indicates that the wind noise reduction performance of the windscreen is not the

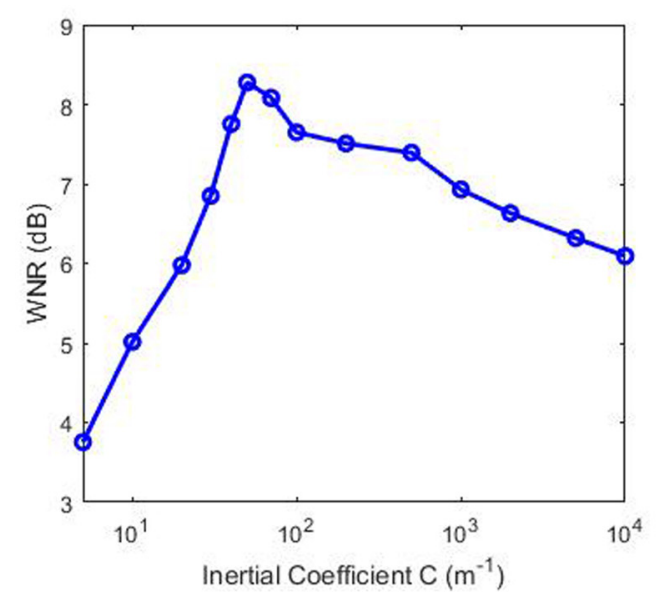

(a)

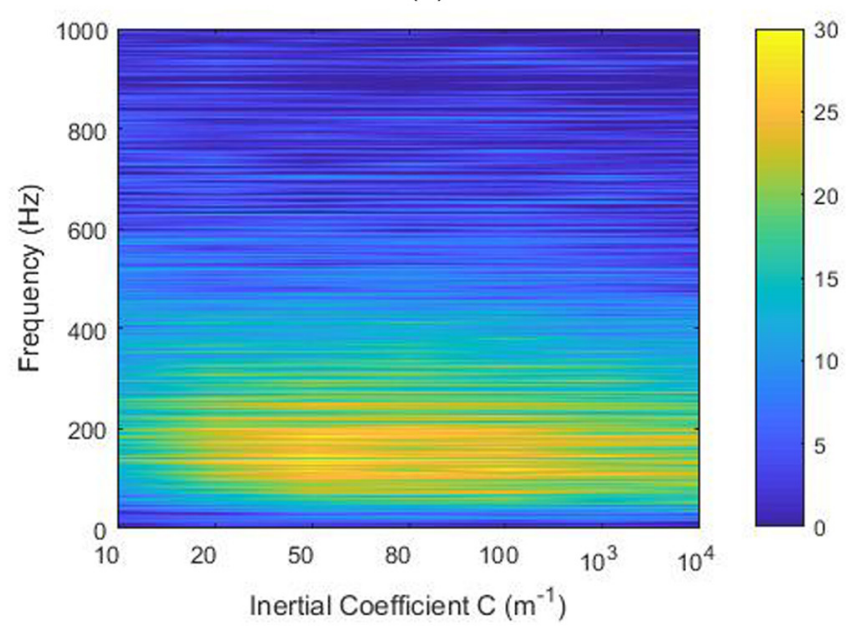

(b)

FIG. 6. (Color online) (a) The overall WNR and (b) the WNR at different frequencies for $90 \mathrm{~mm}$ windscreens with different inertial coefficients at the wind speed $U=4 \mathrm{~m} / \mathrm{s}$.

supposition of the viscous and inertial effect; but in contrast, it is dominated by the larger effect. The WNR is the largest when the viscous and inertial coefficients are approximately $10^{8} \mathrm{~m}^{-2}$ and $50 \mathrm{~m}^{-1}$, respectively, which is denoted by a cross in Fig. 8 . The porous windscreens with larger or smaller viscous and inertial coefficients have inferior performance.

In summary, the above simulation results show that the choice of the porous material for microphone windscreens should take into account both the turbulence suppression inside and the wake generation behind the windscreen, and there exists optimal viscous and inertial coefficients to reach the maximum performance. It is noteworthy that the optimal viscous and inertial coefficients should depend on the diameter of the porous windscreens and the wind speed of the incoming flow, and the values presented in Fig. 8 are only applicable for a $90 \mathrm{~mm}$ spherical porous windscreen at the wind speed $U=4 \mathrm{~m} / \mathrm{s}$.

\section{EXPERIMENTS}

To verify the reliability of the simulations, experiments were performed with a fan in the SIAL sound pod at RMIT 


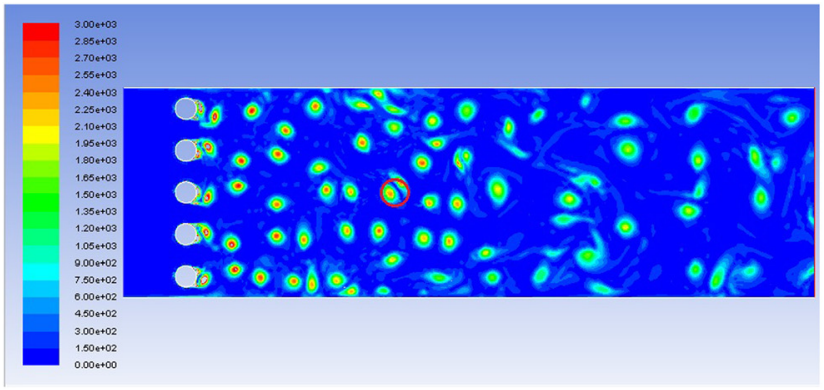

(a)

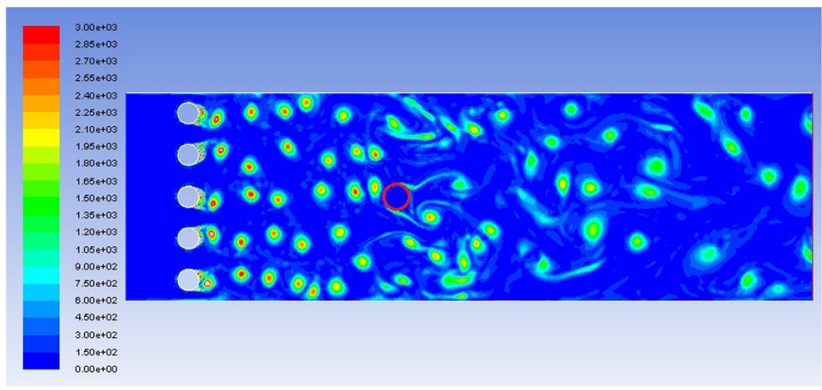

(c)

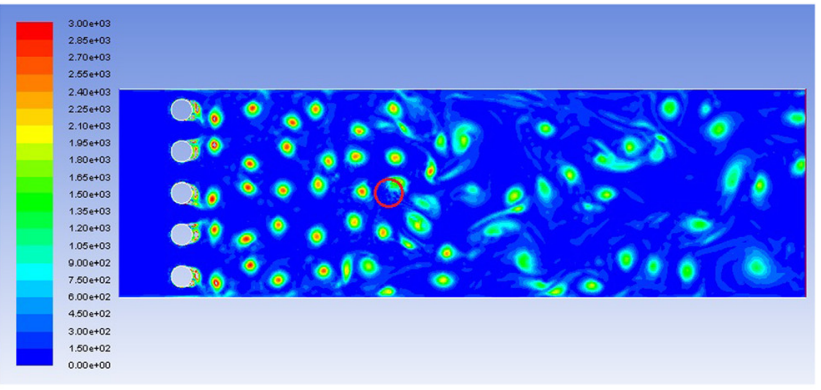

(b)

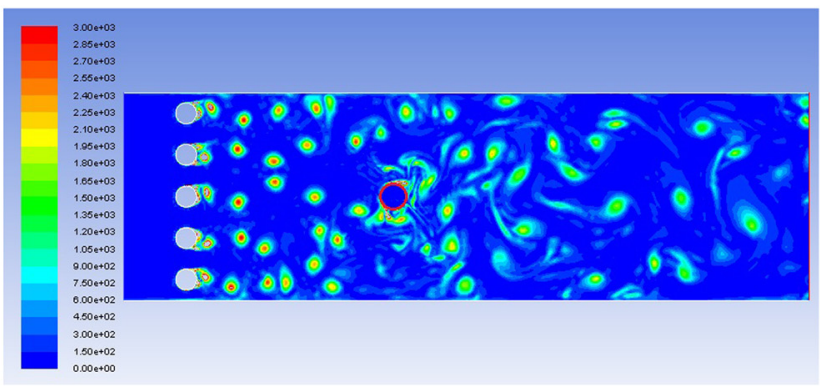

(d)

FIG. 7. (Color online) The vorticity contour for different inertial coefficients, (a) $C=0$ (no windscreen), (b) $C=10 \mathrm{~m}^{-1}$, (c) $C=50 \mathrm{~m}^{-1}$, and (d) $C=100 \mathrm{~m}^{-1} \mathrm{~m}^{-2}$. The red circle at the middle denotes the location of the microphone windscreen.

University, as shown in Fig. 9. The SIAL sound pod is a small room where the walls and floor are treated with sound absorptive material. The fan and the microphone were approximately $0.8 \mathrm{~m}$ above the floor, with a separation distance of $0.5 \mathrm{~m}$. The wind noise was measured with a $\mathrm{B} \& \mathrm{~K}$ type 4189 prepolarized free field $1 / 2$ in. microphone and a G.R.A.S. Type $40 \mathrm{BF} 1 / 4$ in. free field microphone, respectively. The $1 / 2$ in. microphone was connected to the $B \& K$ type 2270 Analyzer via a B\&K Type ZC 0032 Preamplifier. The system was calibrated with a B\&K type 4231 calibrator. The wind noise reduction by 5 spherical porous microphone windscreens with a diameter of $90 \mathrm{~mm}$ were measured in the experiments. The porosity of the porous microphone windscreens varied from 20 to 60 pores per inch (PPI) with a step of 10 PPI, as shown in Fig. 9(c).

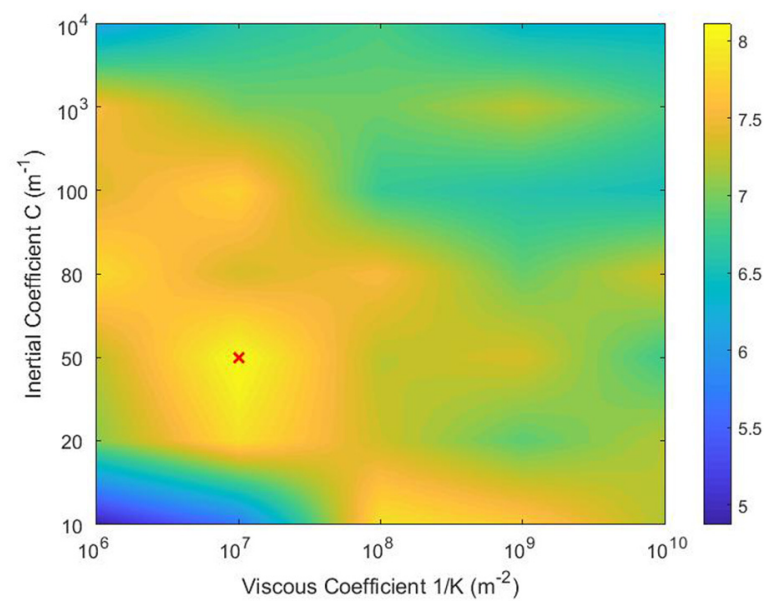

FIG. 8. (Color online) The overall WNR for various viscous and inertial coefficients for a $90 \mathrm{~mm}$ spherical porous windscreen at the wind speed $U=4 \mathrm{~m} / \mathrm{s}$.
In the experiments, the fan ran at its highest speed and the mean wind speed around the microphone was about $4.2 \mathrm{~m} / \mathrm{s}$. The Reynolds number of the flow in the fan tests can be estimated as $\operatorname{Re}=U D / \nu=2.8 \times 10^{4}$ (where $U=4.2 \mathrm{~m} / \mathrm{s}$ is the mean wind speed, $D=0.1 \mathrm{~m}$ is the fan blade length and $\nu=1.511 \times 10^{-5}$ is the air kinematic viscosity at the temperature of $20^{\circ} \mathrm{C}$ ). The Reynolds number based on the Taylor microscale is proportional to the square root of the Reynolds number, i.e., $\operatorname{Re}_{\lambda} \approx(20 \operatorname{Re} / 3)^{1 / 2}=432$ (Pope, 2000). In contrast, the Reynolds number based on the Taylor microscale in the atmosphere $\mathrm{Re}_{\lambda}$ varies from 4250 to 19500 , which is much larger than that in the fan test (Pearson and Antonia, 2001). The length scale of the atmospheric turbulence is much larger than that in the fan test, which might have a significant effect on the wind noise reduction performance of the porous microphone windscreens. This effect is not considered in this paper and will be studied in the future work.

The wind noise was first measured by using the bare microphone inside the air flow and the background noise was measured by placing the microphone out of the flow but at the same distance from the fan. The wind noise and the background noise spectra of the environment with the fun running are compared in Fig. 10(a), which shows that the wind noise level is much higher than the background noise, hence the measurement results with the microphone placed inside the air flow were primarily due to the wind turbulence from the fan. The peak at $62.5 \mathrm{~Hz}$ and its harmonics in the background noise are the mechanical noise due to the fan blade.

The wind noise inside the porous microphone windscreens were measured the same way as that for the bare microphone and the measured wind noise spectra are also shown in Fig. 10(a). The wind noise spectra measured inside 


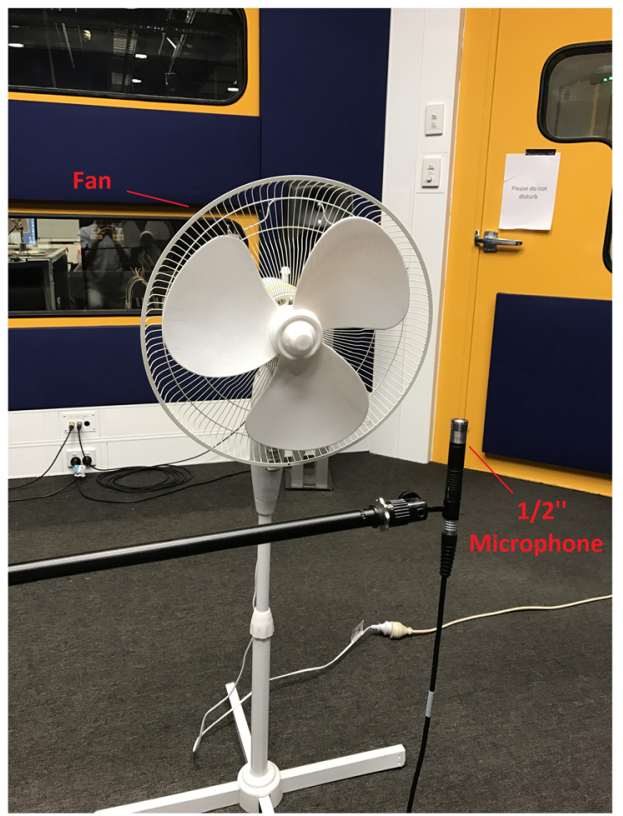

(a)

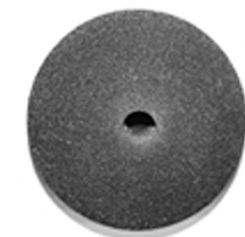

(c)

60 PPI

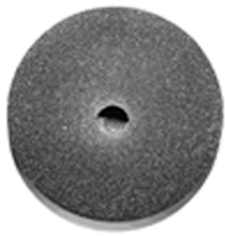

50 PPI

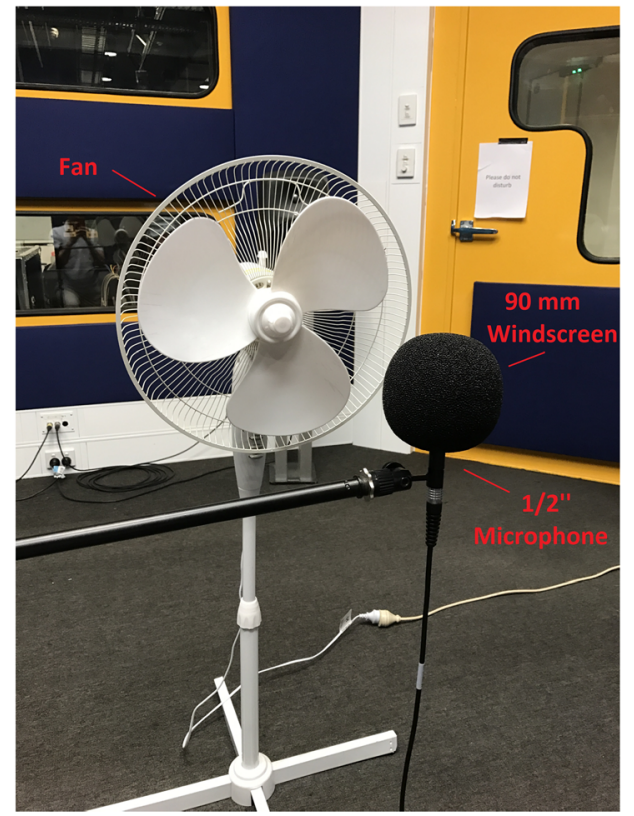

(b)

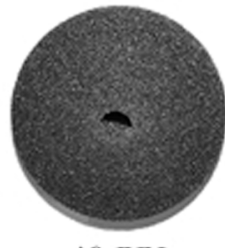

40 PPI

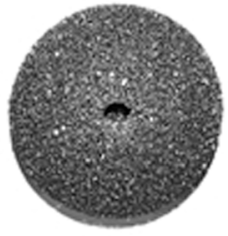

30 PPI

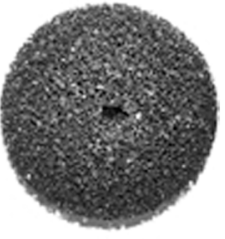

20 PPI
FIG. 9. (Color online) Experimental setup (a) without and (b) with the porous windscreen installed on a $1 / 2$ in. microphone, and (c) the $90 \mathrm{~mm}$ spherical porous microphone windscreens of different porosity from 20 to 60 PPI.
30 and 50 PPI windscreens are not shown for the sake of brevity. It is clear in Fig. 10(a) that the wind noise level inside the porous windscreens is still above the background noise, thus the measurement results are effective. The porous microphone windscreens can attenuate the wind noise due to the turbulence but not the mechanical noise from the fan blade, which justifies the rationality of improving the acoustic measurement accuracy by porous microphone windscreens under windy conditions.

The wind noise spectra measured inside windscreens of varying porosity are almost indistinguishable above $50 \mathrm{~Hz}$ due to the harmonic mechanical noise from the fan blade. However, it can be seen from the spectra below $50 \mathrm{~Hz}$ in Fig. 10(a) that the wind noise inside the $40 \mathrm{PPI}$ windscreen is lower than that inside both the 20 and 60 PPI windscreens, which demonstrates that the existence of an optimal porosity for the porous windscreen to achieve the best performance. This can be more clearly observed from Fig. 10(b), which shows the overall WNR in the broad frequency band from 1 to $1000 \mathrm{~Hz}$ as a function of the PPI values. The overall WNR first increases with the porosity but then decreases after reaching the maximum performance at $40 \mathrm{PPI}$.

As shown in Fig. 9(c), with the increase in the PPI value from 20 to 60 , the number of pores is increased while the pore size is reduced, so the contact surface area between the air flow and the porous frame is increased which leads to the increase in the viscous forces on the air flow (Narasimhan, 2013). Since the viscous coefficient $1 / K$ is a lumped measure of the total viscous forces, it increases correspondingly with the increased PPI value (Narasimhan, 2013). Similarly, the inertial coefficient $C$ also increases with the PPI value because the inertial forces from the porous frame on the air flow increases (Narasimhan, 2013). Both the viscous and inertial coefficients can be measured with hydraulic equipment (Lage et al., 2005). However, the viscous and inertial coefficients of the porous windscreens used in this paper were not measured because no such hydraulic equipment is available to us at present.

In summary, both the viscous and inertial coefficients of the porous windscreens increase with the PPI value although the specific values for the porous microphone windscreens used in our experiments are not known. Therefore the measurement results in Fig. 10 verified the simulation results that there exists an optimal value of viscous and inertial coefficients for porous microphone windscreens to reach the best performance, and the design of the porous microphone windscreens should take into account both the turbulence suppression inside and the wake generation behind the microphone windscreens.

The simulation and experiment results in this paper indicate that the wind noise reduction performance can be further improved if the turbulence inside the windscreens can be suppressed and in the meantime no wake is generated behind the windscreen, which might be achieved with porous microphone windscreens with variable porosity. On the other hand, metamaterials can be explored to manipulate the fluid flow around three-dimensional bodies, e.g., 


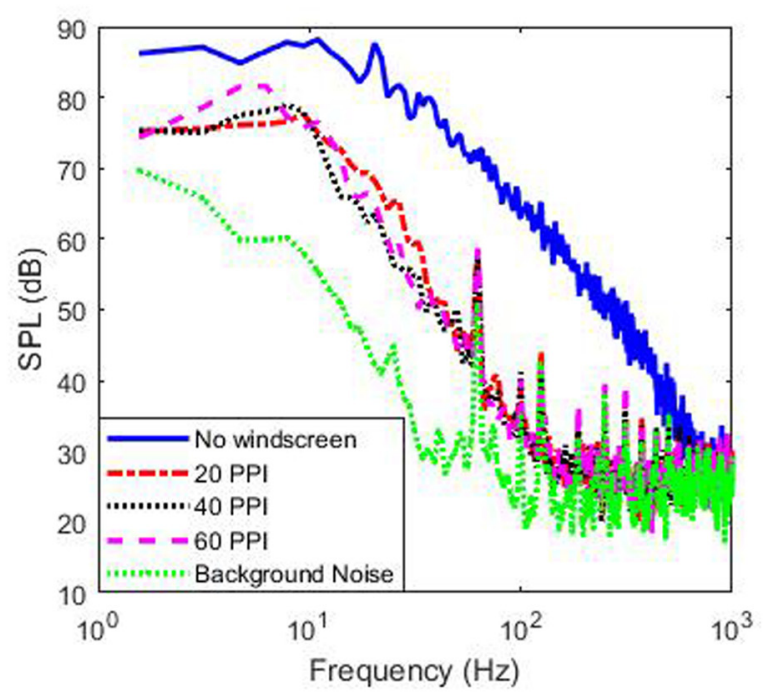

(a)

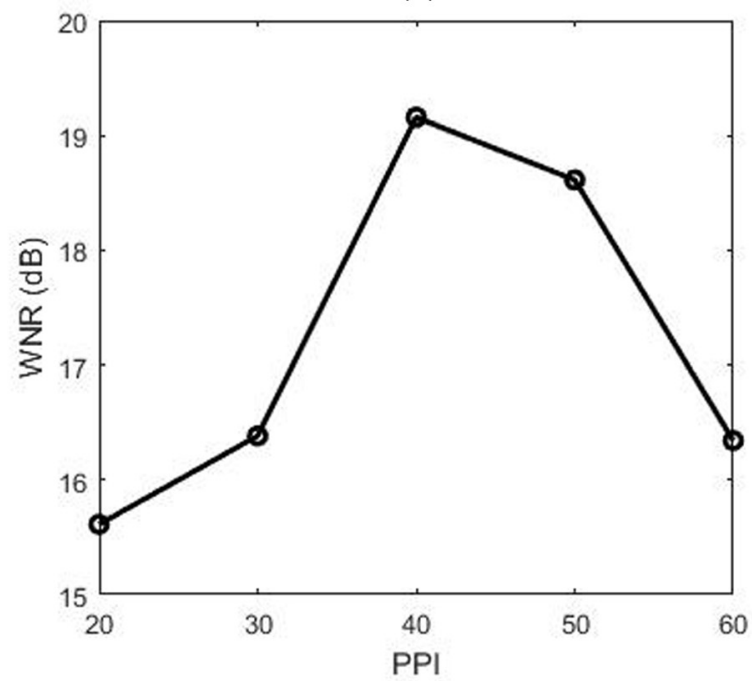

(b)

FIG. 10. (Color online) The measurement results of (a) the wind noise spectra and (b) the overall WNR as a function of the porosity.

Urzhumov and Smith (2011) investigated fluid flow cloak which preserves the flow that would have existed in the absence of the object so that the downstream wake is eliminated. However, their study is based on numerical simulations for the non-turbulent flows, much more work is needed for developing prototype metamaterial microphone windscreen in the future.

\section{CONCLUSIONS}

This paper investigates the physical mechanism of wind noise reduction provided by porous microphone windscreens numerically first, and simulation results indicate that the wind noise reduction first increases and then decreases with both the viscous and inertial coefficients after reaching a maximum. The phenomenon is explained qualitatively and it is shown that the design of the microphone windscreen material could be optimized by compromising the turbulence suppression inside and the wake generation behind the windscreen to reach its maximum performance. In addition, the performance of the porous windscreens with various combined viscous and inertial coefficients is simulated, and results show that the wind noise reduction can be the largest for a certain combination of the viscous and inertial coefficients values (i.e., 1/ $K=10^{8} \mathrm{~m}^{-2}$ and $C=50 \mathrm{~m}^{-1}$ for the simulations in this paper). Experimental results with five porous microphone windscreens with porosity from 20 to 60 PPI show the 40 PPI windscreen to have the best performance and this verifies the simulation results. Future work includes measuring the exact viscous and inertial coefficients of the porous windscreens and studying the effect of the windscreen diameter and shape on the wind noise reduction performance.

\section{ACKNOWLEDGMENTS}

This research was supported under the Australian Research Council's Linkage Project funding scheme (No. LP140100740).

Alamshah, V., Zander, A., and Lenchine, V. (2015). "Effects of turbulent flow characteristics on wind induced noise generation in shielded microphones," Proc. Acoust. 2015, Hunter Valley, Australia, pp. 1-11.

Boersma, H. F. (1997). "Characterization of the natural ambient sound environment: Measurements in open agricultural grassland," J. Acoust. Soc. Am. 101, 2104-2110.

Dawson, M. A., Germaine, J. T., and Gibson, L. J. (2007). "Permeability of open-cell foams under compressive strain," Int. J. Solids Struct. 44, 5133-5145.

Gotoh, T., and Fukayama, D. (2001). "Pressure spectrum in homogeneous turbulence," Phys. Rev. Lett. 86, 3775-3778.

Lage, J. L., Krueger, P. S., and Narasimhan, A. (2005). "Protocol for measuring permeability and form coefficient of porous media," Phys. Fluids 17, $1-4$.

Larsson, M., Johansson, S., Håkansson, L., and Claesson, I. (2005). "Microphone windscreens for turbulent noise suppression when applying active noise control to ducts," Proc. 12th Int. Congr. Sound Vib., Lisbon, Portugal, pp. 1-8.

Lin, I.-C., Hsieh, Y.-R., Shieh, P.-F., Chuang, H.-C., and Chou, L.-C. (2014). "The effect of wind on low frequency noise," Inter. Noise 2014, Melbourne, Australia, pp. 1-12.

Morgan, S., and Raspet, R. (1992). "Investigation of the mechanisms of low-frequency wind noise generation outdoors," J. Acoust. Soc. Am. 92, 1180-1183.

Narasimhan, A. (2013). Essentials of Heat and Fluid Flow in Porous Media (CRC, Boca Raton, FL), pp. 61-105.

Nithiarasu, P., Seetharamu, K. N., and Sundararajan, T. (1997). "Natural convective heat transfer in a fluid saturated variable porosity medium," Int. J. Heat Mass Transf. 40, 3955-3967.

Pearson, B. R., and Antonia, R. A. (2001). "Reynolds-number dependence of turbulent velocity and pressure increments," J. Fluid Mech. 444, 343-382.

Phelps, W. D. (1938). "Microphone wind screening," RCA Rev. 111, 203-212.

Pope, S. B. (2000). Turbulent Flows (Cambridge University Press, New York), $200 \mathrm{pp}$.

Raspet, R., and Webster, J. (2015). "Wind noise under a pine tree canopy," J. Acoust. Soc. Am. 137, 651-659.

Raspet, R., Webster, J., and Dillion, K. (2006). "Framework for wind noise studies," J. Acoust. Soc. Am. 119, 834-843.

Raspet, R., Webster, J., and Naderyan, V. (2014). "Mechanisms for wind noise reduction by a spherical wind screen," J. Acoust. Soc. Am. 135, 2381-2381.

Raspet, R., Yu, J., and Webster, J. (2008). "Low frequency wind noise contributions in measurement microphones," J. Acoust. Soc. Am. 123, $1260-1269$. 
Shams, Q. A., Zuckerwar, A. J., and Sealey, B. S. (2005). "Compact nonporous windscreen for infrasonic measurements," J. Acoust. Soc. Am. 118, $1335-1340$.

Strasberg, M. (1988). "Dimensional analysis of windscreen noise," J. Acoust. Soc. Am. 83, 544-548.

Tsuji, Y., and Ishihara, T. (2003). "Similarity scaling of pressure fluctuation in turbulence," Phys. Rev. E 68, 026309.

Urzhumov, Y. A., and Smith, D. R. (2011). "Fluid flow control with transformation media," Phys. Rev. Lett. 107, 074501.

van den Berg, G. P. (2006). "Wind-induced noise in a screened microphone," J. Acoust. Soc. Am. 119, 824-833.
Wang, L., Zander, A. C., and Lenchine, V. V. (2012). "Measurement of the self-noise of microphone wind shields," Proc. 18th Aust. Fluid Mech. Conf., Launceston, Australia, pp. 1-10.

Xu, Y., Zheng, Z., and Wilson, D. (2011). "A computational study of the effect of windscreen shape and flow resistivity on turbulent wind noise reduction," J. Acoust. Soc. Am. 129, 1740-1747.

Yu, J., Raspet, R., Webster, J., and Abbott, J. (2011). "Wind noise measured at the ground surface," J. Acoust. Soc. Am. 129, 622-632.

Zheng, Z., and Tan, B. (2003). "Reynolds number effects on flow/acoustic mechanisms in spherical windscreens," J. Acoust. Soc. Am. 113, $161-166$. 